\title{
IMPLEMENTATION AND VERIFICATION OF PROGRAMMABLE SECURITY
}

\author{
Stephen Magill, Bradley Skaggs, Mauricio Papa and John Hale
}

\begin{abstract}
This paper presents a methodology for augmenting programming languages with configurable security services. In particular, it describes JPAC, a Java extension that provides syntax for expressing discretionary package-based access control. Access control is based on a variation of a ticket-based authorization model. The authorization model has been succesfully implemented in the Isabelle theorem proving environment. Moreover, a novel cryptographic verification formalism is used to analyze JPAC's secure method invocation protocol. Programmable security architectures and cryptographic protocol verification formalisms used in concert can provide verifiably secure programming systems for Internet applications.
\end{abstract}

Keywords: Formal methods, programmable security, authentication, process calculi

\section{Introduction}

Internet computing is a catalyst for the development of new programming language protection models, security APIs, and software integration and interoperability frameworks. Developers rely on protection models to check code integrity and guard memory boundaries at compile-time and run-time [4, 8]. Together, protection models and security APIs comprise the state of the art for safe-guarding applications running in open, heterogeneous environments. However, these tools do not ensure that a security policy articulated with an API is consistent or viable. Moreover, very little is available to programmatically link elements in a protection model with a security API. As a result, security APIs are commonly used in an ad hoc fashion yielding unpredictable security policies.

Programmable security provides syntactic and semantic constructs in programming languages for coherently embedding security functionality within applications [9]. Developers use special syntax to express security policies 
within code in the same way that types are used to express constraints on variable behavior. This approach facilitates compile-time and run-time securitychecking (analogous to type-checking) to verify that no potential security policy violations exist within a program.

This paper describes the Java Package Access Control (JPAC) System. JPAC extends the Java programming language with syntax for specifying discretionary access control policies at the package level. The JPAC access control scheme is based on a primitive authorization model that has been mechanized in Isabelle, an interactive theorem prover. Access to Java data and code elements under JPAC is fully mediated by a secure method invocation scheme. As this paper demonstrates, both the authorization model and its implementation using the secure method invocation scheme are amenable to formal analysis.

Section II of the paper discusses the broad topic of programmable security. Section III presents our authorization model and illustrates the potential for formal analysis of security policies expressed in the model. Section IV describes the JPAC archtitecture and each of its essential components. Section $\mathrm{V}$ provides a detailed examination of the secure method invocation protocol, and section VI presents a formal analysis of the scheme using a novel cryptographic protocol verification system. Section VII provides conclusions.

\section{Programmable Security}

Object-oriented programming languages employ protection schemes based on classes, variables and methods. Java 1.0 provides packages to group program units (classes and interfaces), creating access boundaries [4]. Java 1.2 lets developers define protection domains to specify sets of classes sharing identical permissions $[7,8]$. The added functionality is given in an API. Wallach et $a l$. propose extensions to the Java security model that employ capabilities and namespace management techniques [24]. Java capabilities are implemented based on the fact that references to objects cannot be fabricated due to Java's type safety features. The disadvantage of these approaches is that no significant compile-time security checking can be performed.

Early work in [5] describes a compile-time mechanism to certify that programs do not violate information flow policies, while [2] provides a flow logic to verify that programs satisfy confinement properties.

In [23], Volpano et al. recast the information flow analysis model in [5] within a type system to establish its soundness. This work led to a sound type system for information flow in a multi-threaded language [20]. JPAC differs in that it promotes a foundational authorization model as a common substrate for various access control schemes [9] to support the static analysis of secure program interoperability. 


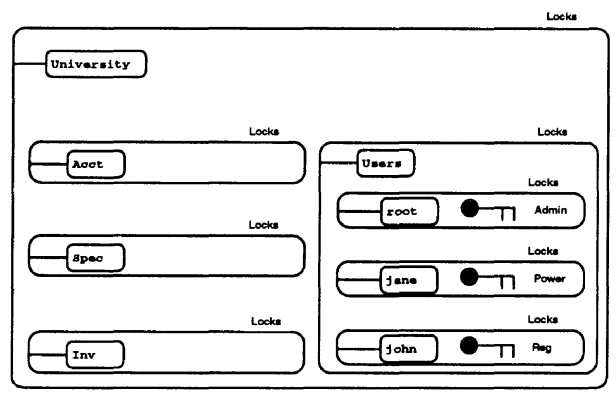

(a) Physical

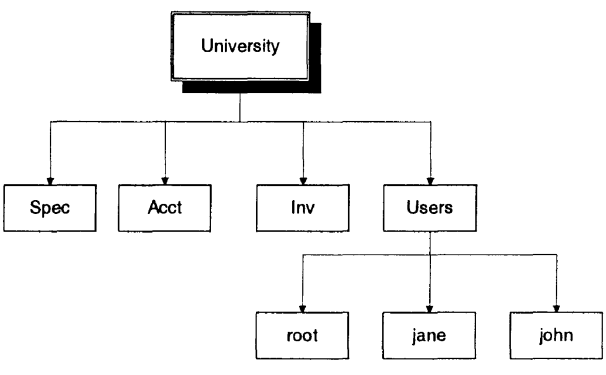

(b) Hierarchical

Figure 1. System views.

Van Doorn et al. extend Modula-3 network objects with security features in [22]. Secure network objects (SNOs) bind programming languages into service for integrating security into objects and methods. The SNO runtime design most closely resembles ours (e.g., using capabilities and access control lists), but the SNO syntax extensions express operational semantics as opposed to policy specifications.

Myers and Liskov describe a decentralized information flow control model in [17]. Myers implemented these ideas in JFlow [18]. While JFlow uses syntactic extensions in a dialect of Java to express information flow, our languages capture more abstract security policies in the discretionary access control domain.

The SLam calculus is a typed $\lambda$-calculus that tracks relevant security properties of programming elements [12]. A compiler that executes static checks enforces type system rules to guarantee program security. While the SLam calculus and our approach are both amenable to static analysis, their formalism relies heavily on secure type systems in functional languages.

\section{Authorization Model}

This section presents a formal semantics for the ticket-based authorization model and a simple scenario illustrating its use and capabilities. A system in our model is represented by a hierarchical structure of node components. Tickets embedded in messages as unforgeable tokens are analogous to capabilities $[13,14]$, conveying privileges of message originators. Message passing only occurs directly between two adjacent object nodes.

To illustrate the concept, consider a University with a transactions system consisting of four databases: Spec, Inv, Acct and Users containing equipment specifications, inventory, account information and users, respectively (see Figure 1a). Furthermore, Users contains a set of three subjects 


\begin{tabular}{|c|c|}
\hline Predicate & Note \\
\hline $\begin{array}{c}\text { Parentso } o_{2}\left(o_{1} \neq o_{2}\right) \\
\text { Adj } s o_{1} o_{2}=\text { Parents } o_{1} o_{2} \vee \text { Parents } o_{2} o_{1} \\
\text { Keys } o_{1} t \\
\text { Lockso } o_{1} o_{2} t\left(\text { Adj } s o_{1} o_{2}\right) \\
\text { Matchs } o_{1} o_{2} o_{3}=\exists t \text { Keys } o_{1} t \wedge \text { Locks } o_{2} o_{3} t \\
\text { Access } s o_{1} o_{1} \\
\text { Access } s o_{1} o_{2} \wedge \text { Matchs } o_{1} o_{2} o_{3} \Rightarrow \text { Access } s o_{1} o_{3}\end{array}$ & $\begin{array}{c}o_{1} \text { is Parent of } o_{2} \text { in state } s \\
o_{1} \text { is adjacent to } o_{2} \text { in state } s \\
o_{1} \text { has } K e y \text { named } t \text { in state } s \\
o_{1} \text { has lock named } t \text { on } o_{2} \text { in state } s \\
\text { Key/Lock matching in state } s \\
\text { Objects have access to themselves } \\
o_{1} \text { has access to } o_{3}\end{array}$ \\
\hline
\end{tabular}

Figure 2. Authorization model.

where each subject possesses a key indicating its access level: root (an administrator with read, write and query permissions), john (a regular user with read permission) and jane (a power user with read and write permission).

Our model consists of rules defining the hierarchy, local access level and access level in the hierarchy as described in the following subsections.

Hierarchy Definition The structure of the hierarchy is represented by a set of predicates of the form Parent $s o_{1} o_{2}\left(o_{1} \neq o_{2}\right)$ [Rule 1], indicating that $o_{1}$ is the parent node of $o_{2}$ in state $s$. Preconditions for the predicates are in parentheses. The hierarchy in our example, as shown in Figure 1b, is represented by the following set of seven predicates:

\begin{tabular}{c|c|c} 
Parent $s$ University Spec & Parent $s$ University Inv & Parent $s$ University Acct \\
Parent $s$ University Users & Parent $s$ Users root & Parent $s$ Users jane \\
Parent $s$ Users john &
\end{tabular}

The adjacency relationships between objects, as required by the messagepassing scheme, can be formally specified with the following predicate [Rule 2]: Adj $s o_{1} o_{2}=$ Parent $s o_{1} o_{2} \vee$ Parent $s o_{2} o_{1}$. This auxiliary predicate is used to establish authorized message flow. Messages can only be directly passed between two adjacent nodes.

Local Access Level Conceptually, tickets represent keys held by subjects that match locks held by objects. Keys are checked for matching object locks to authorize access requests.

Access level in the hierarchy is defined by predicates of the form Key $s o_{1} t$ [Rule 3] and Lock $s o_{1} o_{2} t$ (Adj $\left.s o_{1} o_{2}\right)$ [Rule 4]. At this level, the model mandates that $o_{1}$ and $o_{2}$ be adjacent for $o_{1}$ to hold such a lock. Access to non-adjacent nodes is determined by inferring access level as described in the next subsection. The first predicate is true when $o_{1}$ has a key named $t$ in state $s$ and the second when $o_{1}$ has a lock named $t$ on object $o_{2}$ in state $s$ (and the precondition is satisfied). Container nodes must be given the corresponding locks in order to validate/delegate messages: 


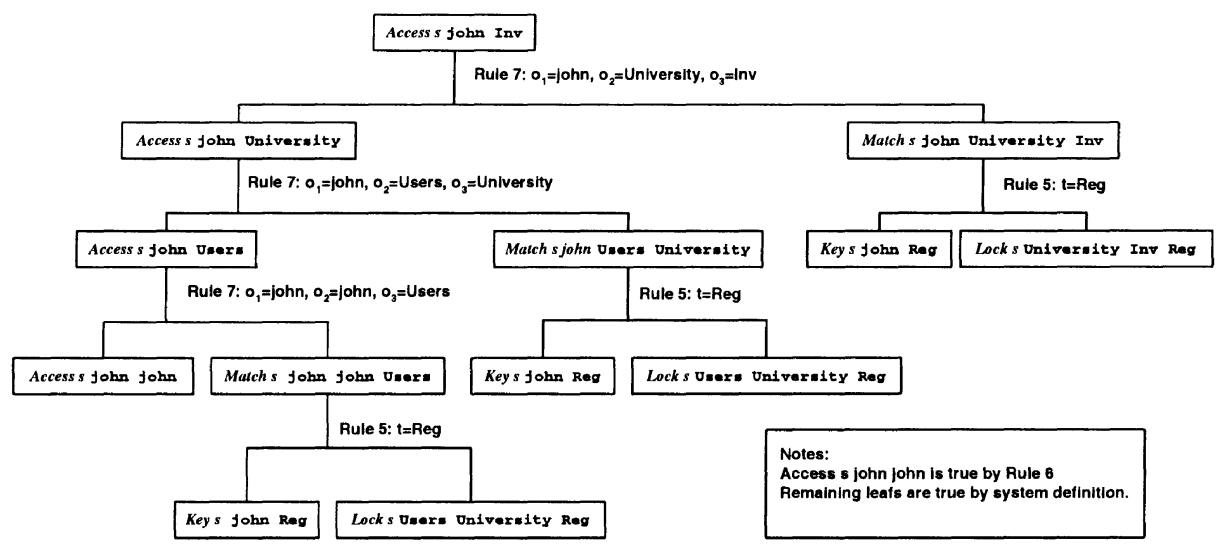

Figure 3. Backward chaining proof.

Lock $s$ University Spec Admin Lock $s$ University Inv Admin Lock $s$ University Inv Reg Lock $s$ University Users Power
Lock $s$ University Spec Power Lock $s$ University Inv Power Lock $s$ University Users Admin Lock $s$ University Users Reg

This embodies the security policy put in place by University. Similarly, a lock structure is defined for Users, having locks on each user corresponding to their access level and locks on University to check messages going up the hierarchy:

Lock $s$ Users root Admin Lock $s$ Users john Reg Lock $s$ Users University Power
Lock $s$ Users jane Power Lock $s$ Users University Admin Lock $s$ Users University Reg

Each user in the hierarchy also possesses locks to check appropriate permissions to send messages through Users:

Lock s root Users Admin | Lock s jane Users Power | Lock s john Users Reg

Finally, each user is given a key matching his/her access level:

Key s root Admin | Key s jane Power | Key s john Reg

Object Access Level Inference We first define a predicate Match, which expresses when a message has permission to be forwarded to $o_{3}$ on behalf of $o_{1}$ in $o_{2}$ (a key/lock matching operation in essence) [Rule 5]:

$$
\text { Match } s o_{1} o_{2} o_{3}=\exists \text { t. Keyso } o_{1} t \wedge \text { Locks } o_{2} o_{3} t .
$$

We define another predicate to represent the ultimate goal of a message. Access $s o_{1} o_{2}$ specifies that $o_{1}$ can access $o_{2}$ from its point of origin in state $s$. Now we can complete the formalization by creating an inductive definition for access between nodes in an object hierarchy with the addition of two rules: 
The consts clauses define types for access methods to the individual components of each of the three datatypes. For instance, OName has type Object $\Rightarrow$ name and returns the name of the object passed to the function. In our Isabelle model, the state $s$ is represented by an Object. Consequently, we must define functions that will determine whether or not an object is part of a given state, i.e. whether or not it is part of the current object structure representing the state. The Object datatype definition is a typical case of nested recursion, it is defined in terms of a list of objects and a list is itself a recursive datatype. Determining membership of an object to the current state, requires the use of two mutually recursive functions: OInStateO and OInStateL. These two functions cover all possible variations of the input.

Having defined the basic datatypes and associated auxiliary functions, the rules in Figure 2 can be easily incorporated into Isabelle (see right-hand side of Figure 4). For instance, Parent 01 O2 State tests whether or not both $\mathrm{O} 1$ and $\mathrm{O} 2$ are in the state and that $\mathrm{O} 2$ is a child of $\mathrm{O} 1$ (by using the membership function defined in the list theory). All other rules are defined similarly with the exception of the Access rule. Access is represented by an inductively defined set of triples. A triple (01, O2, State) is in Access if and only if the triple satisfies the induction rules Base and Ind which correspond to rules 6 and 7 in the authorization model. The theorem ( $j \circ h n$, invt, university): Access in Isabelle corresponds to the example previously proved by hand (Figure 3 ).

The outline for the proof in Figure 3 served as a guide to complete the backward-chaining proof in Isabelle.

\section{Java Package Access Control}

This section presents, a programmable package-based protection scheme for Java (JPAC) using the authorization model presented in the previous section. Standard Java syntax offers four different access level qualifiers for class members with well defined access boundaries: private, protected, public and package. In particular, the public qualifier is the only one that allows a programmer to cross the package boundary. Unfortunately, declaring a member public grants access to the world. JPAC uses minimal syntax extensions to provide developers with discretionary and fine-grained access control for Java applications. Note that JPAC extends, not replaces, the existing Java security architecture. Constructs on the extended grammar are translated into standard Java by means of a specialized preprocessor.

JPAC Grammar Java syntax extensions used to express package-based protection are shown in Figure 5. The EBNF productions in Figure 5 change the way a compilation unit is named by making PackageDeclaration 


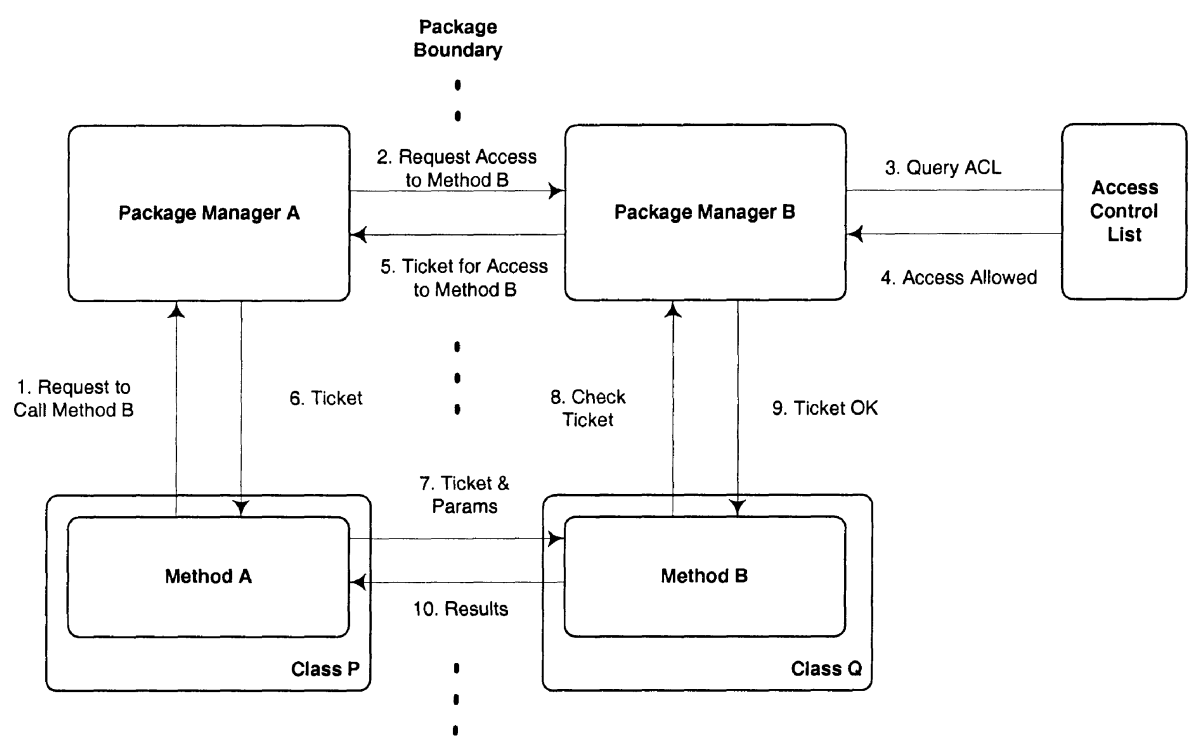

Figure 6. Secure method invocation.

The secure method invocation protocol uses a ticket-based access control scheme to enforce application-level policies at runtime. Authenticated entitities obtain a ticket which can be exchanged for method access. Most of the authentication work rests with package managers, so that methods remain relatively lightweight in JPAC.

An overview of the protocol is provided in Figure 6. The protocol consists of ten communication steps that are functionally divided into two phases: obtaining a ticket from the callee's package manager and the actual method call.

This two-phase scheme has the advantage of reducing system overhead. Once a ticket is obtained, successive calls proceed by skipping the first phase.

\section{Verification}

This section demonstrates the use of a novel cryptographic protocol verification formalism to analyze JPAC's secure method invocation protocol. The formalism uses elements from various process calculi $[1,15,16]$. It combines the convenient modeling properties of process calculi with the powerful reasoning ability of authentication logics, such as BAN [3]. In the analysis process, a subtle flaw in the secure method invocation protocol is discovered and corrected.

Protocol Logic The secure method invocation protocol is formally modeled with a subset of the protocol logic defined in [19]. The logical system is a simple process calculus with pattern-based message exchange and support for 


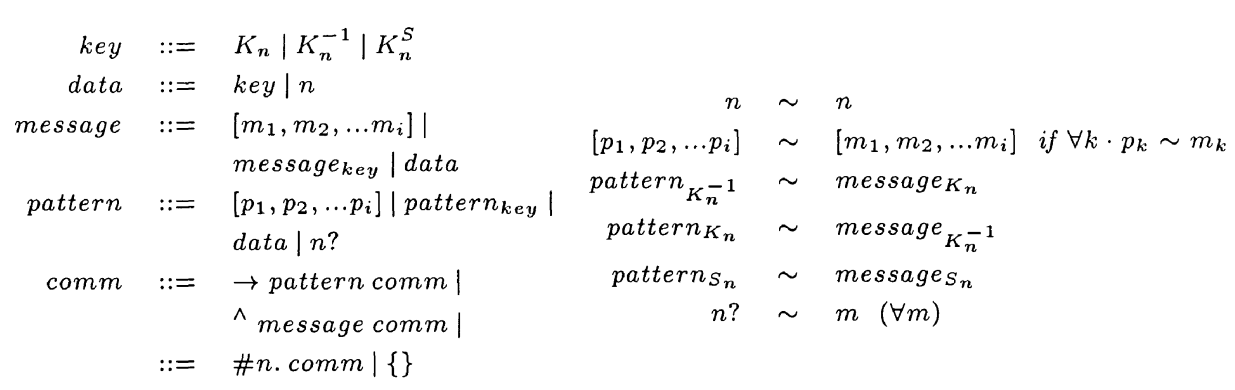

(b) Pattern matching

(a) BNF Grammar

Figure 7. Protocol logic.

both public key and symmetric encryption. The BNF grammar for the process calculus is shown in Figure 7(a).

$n$ denotes a member of an infinite set of names, which represents our data. A key is either public, private, or symmetric. The term "data" is used to encompass keys and names. Messages are strings of data or encrypted messages (message $_{\text {key }}$ denotes a message encrypted under key). Pattern structure mirrors message structure with the addition of a "wildcard" construction ( $n$ ?), which makes $n$ act much like a variable.

comm comprises the set of communication sequences, with output denoted by $\left({ }^{\wedge}\right)$ and input denoted by $(\rightarrow)$. The \#n. comm construction is read as "new name $n$ in comm". It binds $n$ such that $n$ inside comm is different from $n$ outside comm. The result is that $\# n$. comm $\cong \operatorname{comm}\left[n^{\prime} / n\right]$ provided $n^{\prime}$ is not free in the system. A name appearing in the system is bound if it is a wildcard or new name and free otherwise.

An agent is defined by a unique ID and a communication sequence. Agent IDs are considered public knowledge. A system is a group of concurrent agents. Concurrency is denoted by (\&), a commutative operator. Addition or deletion of an agent whose communication is \{\} (the nil agent) also results in a system congruent to the original (a simplification rule).

$$
\begin{aligned}
& \text { agent }:=(n, \text { comm }) \\
& \text { system }::=\text { agent \& system } \mid\{\}
\end{aligned}
$$

Communication occurs when the pattern exposed by one agent matches the message offered by another (pattern matching rules are shown in Figure 7(b)). At this point, the message is transmitted and both agents are said to reduce. The following reduction rules define this behavior:

$$
\text { Out: }:^{\wedge} m a \stackrel{\bar{m}}{\Rightarrow} a \quad \text { In: } \frac{m \sim p}{\rightarrow p a \stackrel{m}{\Rightarrow} a[m / p]} \quad \text { Comm: } \frac{a_{1} \stackrel{\bar{m}}{\Rightarrow} a_{1}^{\prime} a_{2} \stackrel{m}{\Rightarrow} a_{2}^{\prime} a_{1}, a_{2} \in S}{S \Rightarrow S\left[a_{1}^{\prime} / a_{1}\right]\left[a_{2}^{\prime} / a_{2}\right]}
$$


The Out and In rules define agent behavior for an agent offering a message and an agent exposing a pattern respectively. The Comm rule defines agent communication. Operators $\Rightarrow, \stackrel{\bar{m}}{\Rightarrow}$ and $\stackrel{m}{\Rightarrow}$ are used to indicate system reduction, agent reduction with output $m$ and agent reduction with input $m$.

Formal Specification In any run of the secure method invocation protocol, there are four agents participating: the initiator method, initiator package, receiver method and receiver package. The definitions for these four agents will be abstracted over the agent IDs. The convention adopted in the formalism below gives IDs much the same properties as IP addresses. $\mathrm{P}_{\text {enc }}$ can be thought of as the "ticket" which grants access to Method B.

The package manager and the package managed by it are assumed to be running on the same machine. Thus, communication within a package is considered to be secure. However, they are included here for completeness. Since the agent ID is evident from our function naming convention it has been omitted in following definitions; agent $_{n}$ is associated with ID $n$.

$$
\begin{aligned}
& \text { agent }_{m_{A}}\left(m_{A}, m_{B}, A, B\right)=\wedge\left[A, m_{B}, P\right] \rightarrow\left[m_{A}, P_{\text {enc }} ?\right]^{\wedge}\left[m_{B}, P_{\text {enc }}\right] \\
& \rightarrow\left[m_{A}, R_{\text {enc }} ?\right]^{\wedge}\left[A, R_{\text {enc }}\right] \rightarrow\left[m_{A}, R ?\right] \\
& \text { agent }_{m_{B}}\left(m_{A}, m_{B}, A, B\right)=\rightarrow\left[m_{B}, P_{\text {enc }} ?\right]^{\wedge}\left[B, P_{\text {enc }}\right] \rightarrow\left[m_{B}, P ?\right] \\
& { }^{\wedge}\left[B, R, P_{\text {enc }}\right] \rightarrow\left[m_{B}, R_{\text {enc }} ?\right]^{\wedge}\left[m_{A}, R_{e n c}\right] \\
& \operatorname{agent}_{A}\left(m_{A}, m_{B}, A, B\right)=\rightarrow\left[m_{A}, m_{B}, P ?\right]^{\wedge}\left[B, A,\left[m_{B}, P\right]_{K_{A}^{-1}}\right] \rightarrow\left[A,\left[m_{B}, P_{e n c} ?\right]_{K_{A}^{-1}}\right] \\
& \left.\wedge\left[m_{A}, P_{\text {enc }}\right] \rightarrow\left[A,\left[m_{B},[R ?, N ?, P]_{K_{B}}\right]_{K_{A}}\right]^{-1}\right]^{\wedge}\left[m_{A}, R\right] \\
& \operatorname{agent}_{B}\left(m_{A}, m_{B}, A, B\right)=\rightarrow\left[B, A,\left[m_{B}, P ?\right]_{K_{A}}\right] \# N .^{\wedge}\left[A,\left[m_{B},[N, P]_{K_{B}^{-1}}\right]_{K_{A}}\right] \\
& \rightarrow\left[B,[N, P]_{K_{B}}\right]^{\wedge}\left[m_{B}, P\right] \rightarrow\left[B, R,[N, P]_{K_{B}}\right] \\
& { }^{\wedge}\left[m_{B},\left[m_{B},[R, N, P]_{K_{B}^{-1}}\right]_{K_{A}}\right]
\end{aligned}
$$

$\mathrm{R}$ is the result of the method call and $\mathrm{P}$ is the parameter set. Notice that pattern matching is used to check the nonce in agent ${ }_{B}$.

Security and Knowledge Inference Let $\operatorname{CS}\left(S, S^{\prime}\right)$ denote the set of all messages passed during all reductions of $S$ to $S^{\prime}$, hereafter referred to as the communications set of $\left(S, S^{\prime}\right)$.

To analyze the security of a protocol, the formalism must examine what knowledge an intruder could collect during protocol runs and whether this knowledge could be used to gain access to private information. All communications are assumed to be over open channels. This allows the intruder to see every message exchanged in the protocol run. 
$\mathrm{Kn}(M)$, the knowledge gained from the set of messages $M$, is defined as:

$$
\begin{aligned}
& \frac{m \in M}{m \in \operatorname{Kn}(M)} \quad \frac{m, m l \in \operatorname{Kn}(M)}{m:: m l \in \operatorname{Kn}(M)} \\
& \frac{m:: m l \in \operatorname{Kn}(M)}{m \in \operatorname{Kn}(M) \wedge m l \in \operatorname{Kn}(M)} \quad \frac{m \in \operatorname{Kn}(M) \wedge k \in \operatorname{Kn}(M)}{\{m\}_{k} \in \operatorname{Kn}(M)} \\
& \frac{\{m\}_{k_{1}} \in \operatorname{Kn}(M) \wedge k_{2} \in \operatorname{Kn}(M) \wedge k_{1} \sim k_{2}}{m \in \operatorname{Kn}(M)}
\end{aligned}
$$

where $k_{1} \sim k_{2}$ means " $k_{1}$ matches $k_{2}$ " in the sense that a public key matches its corresponding private key and two symmetric keys match each other if they are the same key. The intruder's knowledge after the system changes from state $S$ to state $S^{\prime}$ then becomes $\operatorname{Kn}\left(C S\left(S, S^{\prime}\right)\right)$.

It is now possible to define the meaning of a secure system. Let:

$T(S)=\left\{\left(S, S^{\prime}\right) \mid S \stackrel{*}{\Rightarrow} S^{\prime}\right\}, S+I=S \cup\{\wedge m \mid m \in \operatorname{Kn}(C S(T(S)))\} \cup$ $\{\rightarrow n$ ? $\}$ and secure $\left(S, \mathrm{M}_{\mathrm{p}}\right)=\nexists m \in \mathrm{Kn}(\mathrm{CS}(\mathrm{T}(\mathrm{S}+\mathrm{I}))) \cdot m \in \mathrm{M}_{\mathrm{p}}$ where $\mathrm{M}_{\mathrm{p}}=$ set of messages that should remain private

A system is secure if the intruder cannot gain access to elements in $M_{p}$. The use of S + I in the definition of "secure" acknowledges that the intruder can take an active approach and insert or remove messages from the communications channel.

Protocol security can now be characterized. A protocol is a set of functions of type $I \rightarrow$ agent where $I=\prod_{\alpha} I D s . \alpha$ is a finite indexing set and $I D s$ is the subset of names that represent agent IDs. Given a protocol, $P$, security of this protocol with respect to a set of private messages is defined as:

$$
\text { Let } \mathrm{S}(\mathrm{P})=\bigcup p(I)(p \in P), \text { secure }\left(P, M_{p}\right)=\operatorname{secure}\left(S(P), M_{p}\right)
$$

$\mathrm{S}$ is the union of the co-domains of the protocol functions. The security property for protocols, as given above, states that a protocol is secure if all the states that are reachable using the communication patterns defined by that protocol are secure. Despite the apparent complexity of the task, a relatively straightforward inductive approach suffices as a proof strategy. The base case consists of a system whose only requirements are that every agent is an application of a protocol function and every element of $M_{p}$ is present in the system. It eventually must be shown that:

$$
\forall \mathrm{p} \in \mathrm{P} \cdot \operatorname{secure}\left(S, M_{p}\right) \Longrightarrow \text { secure }\left(p(I) \& S, M_{p}\right)
$$

In our analysis we consider concurrent runs of a protocol and the intruder's ability to communicate with other agents. As a rule, we keep track of the intruder's knowledge $\left(\mathrm{Kn}_{\mathrm{I}}\right)$ at all times. If it is learned that a particular protocol function adds to $\mathrm{Kn}_{\mathrm{I}} \cap K n\left(M_{p}\right)$, then that line of communications is followed, as it may lead to the discovery of a security vulnerability. 
Protocol Analysis This approach has been used to reveal and correct a flaw in the secure method invocation protocol. Since the goal of the protocol is that only an authorized methods get results of a method call, our goal was to show that the protocol is secure with respect to the method result. Initial analysis showed that an intruder could access method results by using a package manager as an oracle.

As a result, the final version of the protocol includes a nonce generated by the caller to prevent the intruder from gaining access to method results unless it is authorized. Based on this analysis, the new protocol is secure.

\section{Conclusions}

Programmable security and programmable access control allow developers to express verifiable protection policies in their applications. JPAC extends the Java language with syntax for expressing package-level discretionary policies. JPAC classes and interfaces can be seamlessly integrated within native Java applications, allowing developers to customize protection policies for selected software components.

A dual complement of formal methods has been used to support the design of JPAC's authorization model and runtime access control mechanisms. The ticket-based authorization model was mechanized in a simple logic using Isabelle to demonstrate the potential for static and dynamic access control policy checking. JPAC's secure method invocation scheme was subjected to formal analysis via a cryptographic protocol verification system, leading to the discovery and elimination of a flaw in the original protocol. The collective success of these two efforts strongly suggests an expansive role for formal methods and tools in the development of programmable security infrastructures.

\section{Acknowledgments}

This research was supported by MPO Contract MDA 904-98-C-A900 and NSF Grant CCR-9984774.

\section{References}

[1] Abadi, M. and Gordon D., Reasoning about cryptographic protocols in the Spi calculus, Proceedings of the Fourth ACM Conference on Computer and Communications Security, pp. 36-47, 1997.

[2] Andrews, G. and Reitman, R., An axiomatic approach to information flow in programs, ACM Transactions on Programming Languages and Systems, vol. 2(1), pp. 56-76, 1980.

[3] Burrows, M., Abadi, M. and Needham, R., A logic of authentication, ACM Transactions on Computer Systems, vol. 8(1), pp. 18-36, 1990. 
[4] Dean, D., Felten, E. and Wallach, D., Java security: ¿From HotJava to Netscape and beyond, Proceedings of the IEEE Symposium on Research in Security and Privacy, pp. 190-200, 1996.

[5] Denning, D. and Denning, P., Certification of programs for secure information flow, Communications of the ACM, vol. 20(7), pp. 504-513, 1977.

[6] Gilgor, V., Huskamp, J., Welke, S., Linn, C. and Mayfield, W., Traditional capability based systems: An analysis of their ability to meet the trusted computer security evaluation criteria, IDA Paper P-1935, Institute for Defense Analyses, Alexandria, Virginia, 1987.

[7] Gong, L., Mueller, M., Prafullchandra, H. and Schemers, R., Going beyond the sandbox: An overview of the new security architecture in the Java Development Kit 1.2, Proceedings of the USENIX Symposium on Internet Technologies and Systems, pp. 103-112, 1997.

[8] Gong, L. and Schemers, R., Implementing protection domains in the Java Development Kit 1.2, Proceedings of the Internet Society Symposium on Network and Distributed System Security, pp. 125-134, 1998.

[9] Hale, J., Threet, J. and Shenoi, S., Capability-based primitives for access control in object-oriented systems, in Database Security, XI: Status and Prospects (eds. T.Y. Lin and X. Qian), Chapman and Hall, London, pp. 134-150, 1998.

[10] Hale, J., Papa, M. and Shenoi, S., Programmable security for objectoriented systems, in Database Security, XII: Status and Prospects (ed. S. Jajodia), Kluwer, Dordrecht, The Netherlands, pp. 109-126, 1999.

[11] Hale, J., Threet, J. and Shenoi, S., A ticket based access control architecture for object systems, Journal of Computer Security, vol. 8, pp. 43-65, 2000.

[12] Heintze, N. and Riecke, J., The SLam calculus: Programming with security and integrity, Proceedings of the Twenty-Fifth ACM Symposium on Principles of Programming Languages, pp. 365-377, 1998.

[13] Karger, P., An augmented capability architecture to support lattice security, Proceedings of the IEEE Symposium on Research in Security and Privacy, pp. 2-12, 1984.

[14] Karger, P., Implementing commercial data integrity with secure capabilities, Proceedings of the IEEE Symposium on Research in Security and Privacy, pp. 130-139, 1988.

[15] Milner, R., Communication and Concurrency, Prentice-Hall, New York, 1989.

[16] Milner, R., Parrow, J. and Walker, D., A calculus of mobile processes, Technical Report ECS-LFCS-89-85\&86, University of Edinburgh, Edinburgh, U.K., 1989. 
[17] Myers, A. and Liskov, B., A decentralized model for information flow control, Proceedings of the Sixteenth ACM Symposium on Operating System Principles, pp. 129-142, 1997.

[18] Myers, A., JFlow: Practical mostly-static information flow control, Proceedings of the Twenty-Sixth ACM Symposium on Principles of Programming Languages, pp. 229-241, 1999.

[19] Papa, M., Bremer, O., Magill, S., Hale, J. and Shenoi, S., Simulation and analysis of cryptographic protocols, in Data and Applications Security: Developments and Directions (eds. B. Thuraisingham, R. van de Riet, K. Dittrich and Z. Tari), Kluwer, Dordrecht, The Netherlands, pp. 89-100, 2001.

[20] Smith, G. and Volpano, D., Secure information flow in a multi-threaded imperative language, Proceedings of the Twenty-Fifth ACM Symposium on Principles of Programming Languages, pp. 355-364, 1998.

[21] Sun Microsystems, Clarifications and amendments to the Java language specification, www.java.sun.com/docs/books/jls/clarify.html, 1999.

[22] Van Doorn, L., Abadi, M., Burrows, M. and Wobber, E., Secure network objects, Proceedings of the IEEE Symposium on Research in Security and Privacy, pp. 211-221, 1996.

[23] Volpano, D., Smith, G. and Irvine, C., A sound type system for secure flow analysis, Journal of Computer Security, vol. 4(3), pp. 167-187, 1996.

[24] Wallach, D., Balfanz, D., Dean, D. and Felten, E., Extensible security architectures for Java, Proceedings of the Sixteenth Symposium on Operating Systems Principles, pp. 116-128, 1997. 